\title{
Die Prüfung von Teilrevisionsinitiativen auf die Verfassungswürdigkeit ihres Inhalts
}

Felix H. Thomann *

Abstimmungen über Initiativen auf Teilrevision der BV werden seit 45 Jahren immer häufiger, weil die nötige Unterschriftenzahl längst zu niedrig ist und das Parlament nur die Einhaltung von Art. 139 Abs. 3 BV prüft. Die Belastung von Bundesbehörden und Stimmberechtigten durch die Behandlung nicht verfassungswürdiger Volksbegehren, welche häufig in erster Linie der politischen Propaganda dienen, und die schleichende Verschiebung der verfassungsmässigen Kompetenzordnung durch Initiativen mit unterstufigem Inhalt werden zu einer Gefahr für das politische Leben der Schweiz. Abhilfe schaffen könnte die Erweiterung der Prüfung von Initiativen auf die Verfassungswürdigkeit ihres Inhalts; sie wäre nach Meinung des Verfassers gemäss Art. 139 BV zulässig und geboten.

I. Einleitung 91

II. Die Bestimmungen der Bundesverfassung über die Teilrevisionsinitiative von 1891 bis heute 92

III. Versuche zur Einführung der Gesetzesinitiative im Bund .............................93

IV. Die Praxis der Bundesversammlung bei der Prüfung von Volksbegehren ........ 95

V. Die Konsequenzen der bisherigen Prüfungspraxis in neuerer Zeit...................98

VI. Die Verschiebung der verfassungsmässigen Kompetenzordnung ....................100

VII. Einwände gegen die Prüfung auf Verfassungswürdigkeit ............................ 107

VIII. Derogierendes Gewohnheitsrecht durch langjährige Prüfungspraxis?. 110

IX. Neueste Entwicklungen .......................................................................113

X. Zuständigkeit zur Prüfung von Volksbegehren............................................114

XI. Zusammenfassung und Ausblick ............................................................115

Zitiervorschlag: Felix H. Thomann, Die Prüfung von Teilrevisionsinitiativen auf die Verfassungswürdigkeit ihres Inhalts in: sui-generis 2015, S. 90

URL: $\quad$ sui-generis.ch/15

DOI: $\quad$ https://doi.org/10.21257/sg.15

* Dr. iur., Rechtsanwalt, Basel.

Dieses Werk ist lizenziert unter einer Creative Commons Namensnennung - Weitergabe unter gleichen Bedingungen 4.o International Lizenz. 


\section{Einleitung}

1 In den letzten 45 Jahren hat die Anzahl eingereichter Volksinitiativen auf Bundesebene stetig zugenommen. Die Initiativenflut - so geht die Klage - belaste Parlament, Regierung und Verwaltung über Gebühr und drohe die Stimmberechtigten zu überfordern ${ }^{1}$. Die Hauptgründe für diese Entwicklung liegen auf der Hand:

- Die für Initiativen nötige Unterschriftenzahl (100'000) ist im Verhältnis zur Anzahl der Stimmberechtigten und in Anbetracht der heutigen Möglichkeiten für die Unterschriftensammlung schon lange zu tief ${ }^{2}$, selbst wenn man in Rechnung stellt, dass seit der Einführung der Teilrevisionsinitiative die Bundeskompetenzen stark ausgedehnt wurden und die Gesetzgebungstätigkeit auf Bundesebene dementsprechend zugenommen hat3.

\footnotetext{
Die Klage ist keineswegs neu (vgl. z.B. Botschaft vom 9. Juni 1975 über eine Erhöhung der Unterschriftenzahlen für Initiative und Referendum, BB1 1975 II 129 ff., 134, 137, zit. Botschaft Erhöhung; Botschaft vom 20. November 1996 über eine neue Bundesverfassung, BBl 1997 I 1 ff., 436 ff., zit. Botschaft BV); sie war aber noch nie so berechtigt wie heute.

2 Die bei Einführung der Initiative auf Teilrevision im Jahre 1891 gültige Unterschriftenzahl von 50 '000 machte damals rund 7,8\% der Stimmberechtigten aus; sie wurde seither einmal (nach der Einführung des Frauenstimmrechts) erhöht und entsprach Ende 2014 nur noch rund 1,9\% der Stimmberechtigten.

3 Die Ausweitung der Bundeskompetenzen und die davon herrührende Zunahme der Bundesgesetzgebung wären im Falle einer Erhöhung der Unterschriftenzahlen für Initiative und Referendum angemessen zu berücksichtigen (vgl. dazu Botschaft BV 448 ff.; Stellungnahme BR vom 15. Juni 2001 zur Parlamentarischen Initiative (Kommission 96.091 SR) Beseitigung von Mängeln der Volksrechte, BBl 2001 6080). A.M. Ch. Hofer: Überlegungen zu Möglichkeiten einer Optimierung der Volksinitiative, in: Jusletter 26. April 2010, Rz 49,
}

- Die Praxis der Bundesversammlung bei der Prüfung von Volksbegehren war bisher zu locker.

2 Beide Faktoren erleichtern den Einsatz von Volksbegehren für sachfremde Zwecke - insbesondere als Mittel der politischen Propaganda - massiv.

3 Die Bundesversammlung hat seit jeher als gegeben angenommen, ihre Kompetenz zur Prüfung von Volksbegehren sei auf die in der Verfassung ausdrücklich als Ungültigkeitsgründe genannten Punkte (heute in Art. 139 Abs. 3 BV: Einheit der Form, Einheit der Materie, Verletzung zwingender Bestimmungen des Völkerrechts) beschränkt. Als Folge dieser Praxis wurde die Bundesverfassung von $1874^{4}$ im Laufe der Zeit von unzähligen Normen überwuchert, deren Platz richtigerweise in einem Gesetz oder in einzelnen Fällen sogar in einer Verordnung gewesen wäre5. Die Totalrevision der Bundesverfassung von 1999 verfolgte u.a. den Zweck, das Grundgesetz von derartigem Ballast zu befreien ${ }^{6}$. Dies gelang auch einigermassen, doch die Entwicklung seit dem Inkrafttreten der revidierten Verfassung lässt wieder den früheren Missstand erkennen: Allzu viele Volksbegehren haben im Verfassungswald nicht Bäume, sondern Unterholz und Unkraut wachsen lassen7.

der für eine proportionale Anhebung der Unterschriftenzahl eintritt.

4 Die Bundesverfassung von 1874 wird im Folgenden auch mit «aBV» bezeichnet.

5 Vgl. dazu Botschaft BV 439.

6 Botschaft BV 42 ff.; Zusammenfassung der Resultate der Beratungen (Bundesversammlung, Link: Zusammenfassung).

7 A. Kley weist in Biaggini et al., Staatsrecht, 2011 (zit. Biaggini et al.), § $24 \mathrm{Rz} 65$ darauf hin, dass die Anreicherung der BV mit Details auch noch andere Gründe hat als Volksbegehren mit nicht verfas- 
4 Die Einleitung einer Verfassungsrevision zur Erhöhung der Unterschriftenzahlen oder zur Erweiterung der in der Bundesverfassung ausdrücklich genannten Ungültigkeitsgründe ist politisch ein heisses Eisen, und der Wille der politischen Behörden, dieses Eisen anzufassen, hält sich zurzeit in engen Grenzen. Umso wichtiger erscheint deshalb die Frage, ob es nach dem geltenden Recht, d.h. ohne Verfassungsrevision zulässig wäre, die Initiativflut durch die Erweiterung der Prüfung von Volksbegehren auf die Verfassungswürdigkeit ${ }^{8}$ ihres Inhalts einzudämmen. Diese Frage wird im Folgenden untersucht.

\section{Die Bestimmungen der Bundesver- fassung über die Teilrevisionsiniti- ative von 1891 bis heute}

5 Die Bundesverfassung von 1874 sah in ihrem dritten Abschnitt (Überschrift: Revision der Bundesverfassung) nur eine Totalrevisionsinitiative vor (Art. 118-121 aBV). Die Einführung der Initiative auf Teilrevision der Bundesverfassung wurde von Volk und Ständen am 5. Juli 1891 beschlossen; die Absätze 1-4 des revidierten Art. $121 \mathrm{aBV}$, welcher Teil des dritten Abschnitts «Revision der Bundesverfassung» bildete, lauteten fortan wie folgt 9 :

sungswürdigem Inhalt.

8 L. Wildhaber, in: J.-F. Aubert et al. (Hrsg.), Kommentar zur Bundesverfassung der schweizerischen Eidgenossenschaft, 1987-1996 (zit. Komm. aBV), Rz 90 zu Art. 118 aBV, verwendet den Terminus «Verfassungswesentlichkeit».

9 Botschaft vom 13. Juni 1890 über die Revision des III. Abschnittes der Bundesverfassung, handelnd von der Revision dieser letzteren, BBl 1890 III 455 ff., zit. Botschaft 1890; BBl 1891 IV 1 ff. Zur Geschichte der Revision vgl. A. Kölz: Neuere Schweizerische Verfassungsgeschichte - Ihre Grundlinien in Bund und Kantonen seit 1848, 2004, 637 ff. (zit. Kölz, NSVG II).
«Die Partialrevision kann sowohl auf dem Wege der Volksanregung (Initiative) als der Bundesgesetzgebung vorgenommen werden.

Die Volksanregung umfasst das von 50 'ooo stimmberechtigten Schweizerbürgern gestellte Begehren auf Erlass, Aufhebung oder Abänderung bestimmter Artikel der Bundesverfassung.

Wenn auf dem Wege der Volksanregung mehrere verschiedene Materien zur Revision oder zur Aufnahme in die Bundesverfassung vorgeschlagen werden, so hat jede derselben den Gegenstand eines besonderen Initiativbegehrens zu bilden.

Die Initiativbegehren können in der Form der allgemeinen Anregung oder des ausgearbeiteten Entwurfes gestellt werden.»

6 Bis zum Inkrafttreten der Bundesverfassung vom 19. April 1999 erfuhr Art. 121 aBV nur eine einzige Änderung: Nach der Einführung des Frauenstimmrechts beschlossen Volk und Stände am 25. September 1977, die Unterschriftenzahl (Abs. 2) von 50'000 auf 100'00o zu erhöhen ${ }^{10}$.

7 Der Verfassungsentwurf des Bundesrates vom 20. November 1996 enthielt die Be-stim-mungen über die Teilrevisionsinitiative im 4. Titel (Volk und Stände) in Art. 129 ${ }^{11}$. Die Bestimmung trug den Titel «Volksinitiative auf Teilrevision der Bundesverfassung»; die Absätze 1-3 lauteten wie folgt:

«10o'ooo Stimmberechtigte können ei-

10 Botschaft Erhöhung 129 ff.; BBl 1977 III 842, 847.

11 Botschaft BV 618. 
ne Teilrevision der Bundesverfassung verlangen.

Die Volksinitiative auf Teilrevision der Bundesverfassung kann die Form der allgemeinen Anregung oder des ausgearbeiteten Entwurfs haben.

Verletzt die Initiative die Einheit der Form, die Einheit der Materie oder zwingende Bestimmungen des Völkerrechts, so erklärt die Bundesversammlung sie für ungültig.»

8 Der Text der Absätze 1-3 wurde in der parlamentarischen Beratung nur durch die Hinzufügung der Worte «ganz oder teilweise» in Abs. 3 abgeändert.

9 In der am 18. April 1999 angenommenen Bundesverfassung steht der Artikel 139, welcher die Volksinitiative auf Teilrevision regelt, im 4. Titel (Volk und Stände) ${ }^{12}$.

Am 9. Februar 2003 wurde Art. 139 BV im Zusammenhang mit der geplanten Einführung der allgemeinen Volksinitiative (Art. 139a BV) abgeändert: Der Titel lautete nunmehr «Formulierte Volksinitiative auf Teilrevision der Bundesverfassung»; der Abs. 2 der Fassung von 1999 wurde gestrichen, und die Absätze 1-2 erhielten die folgende Formulierung ${ }^{13}$ :

"10o'ooo Stimmberechtigte können innert 18 Monaten seit der amtlichen Veröffentlichung ihrer Initiative eine Teilrevision der Bundesverfassung verlangen.

Verletzt die Initiative die Einheit der

12 AS 19992556 ff.; Bestimmungen über die Volksinitiative enthalten auch die Art. 193 und 194 BV im 6. Titel «Revision der Bundesverfassung und Übergangsbestimmungen».

13 BB1 2002 6485; 20033111.
Form, die Einheit der Materie oder zwingende Bestimmungen des Völkerrechts, so erklärt die Bundesversammlung sie für ganz oder teilweise ungültig.»

11 Am 27. September 2009 wurden die nie in Kraft getretenen Bestimmungen über die allgemeine Volksinitiative wieder aus der Verfassung gestrichen, weil sich die Ausführungsgesetzgebung als «zu komplex» erwiesen hatte ${ }^{14}$. Der Art. 139 BV erhielt wieder die Form, die am 18. April 1999 beschlossen worden war ${ }^{15}$.

12 Die Bundesverfassung sah somit von 1891 an bis heute Volksbegehren immer ausdrücklich nur als Verfassungsinitiativen vor; auf die Bestrebungen zur Einführung der Gesetzesinitiative ist unter III. näher einzugehen.

\section{Versuche zur Einführung der Ge- setzesinitiative im Bund}

13 Die Einführung der Gesetzesinitiative bzw. einer «allgemeinen» Initiative auf Bundesebene war im Lauf der Jahrzehnte wiederholt Gegenstand der politischen Diskussion ${ }^{16}$ :

1. Schon mit der Vorlage von 1872 zur Totalrevision der Bundesverfassung von 1848 hatte die Bundesversammlung die Gesetzesinitiative einführen

14 U. Häfelin, W. Haller, H. Keller: Schweizerisches Bundesstaatsrecht, 8. Auflage 2012 Rz 1798-1799, zit. Häfelin/Haller/Keller. Auf den Ablauf der Verfassungsänderungen von 2003 und 2009 wird unter III. noch zurückzukommen sein.

15 BB1 2009 13, 8722.

16 Die folgenden Ausführungen gehen nicht auf persönliche Vorstösse von Parlamentariern und auf parlamentarische Initiativen ein (vgl. dazu insbesondere BBl 1960 I 362 f. und L. Wildhaber, Komm. aBV, Rz 101 zu Art. 118). 
wollen ${ }^{17}$. Volk und Stände lehnten die Vorlage jedoch am 12. Mai $1872 \mathrm{ab}^{18}$. In der bundesrätlichen Totalrevisionsvorlage von 1873 war die Gesetzesinitiative ebenfalls enthalten ${ }^{19}$; sie wurde aber durch das Parlament gestrichen und bildete deshalb nicht Teil des endgültigen Textes, der von Volk und Ständen angenommen wurde ${ }^{20}$.

2. 1906 kam die Einführung der Gesetzesinitiative erneut vor die Bundesversammlung. Dem Projekt war jedoch kein Erfolg beschieden: Der Na-

Im Entwurf des Bundesrates war die Gesetzesinitiative noch nicht enthalten gewesen (BBl 1870 II 708).

Art. 89 des Verfassungsentwurfs vom 5. März 1872 lautete:

«Wenn 50'000 stimmberechtigte Bürger oder fünf Kantone die Abänderung oder Aufhebung eines bestehenden Bundesgesetzes oder eines Bundesbeschlusses, oder über eine bestimmte Materie die Erlassung eines neuen Bundesgesetzes oder Bundesbeschlusses anbegehren, und diesem Begehren nicht vertragsrechtliche Verpflichtungen des Bundes entgegenstehen, so haben die beiden Räthe, wenn sie dem Begehren zustimmen, den einschlägigen neuen Gesetz- oder Beschlussvorschlag zu vereinbaren und dem Volke zur Annahme oder Verwerfung vorzulegen.

Stimmen nicht beide Räthe dem Begehren zu, so ist dasselbe der Abstimmung des Volkes zu unterstellen, und wenn die Mehrheit der stimmenden Bürger sich dafür ausspricht, so haben die Räthe einen entsprechenden Gesetz- oder Beschlussvorschlag aufzustellen und dem Volke zur Annahme oder Verwerfung vorzulegen.»

Der Text der Verfassungsvorlage vom 5. März 1872 wurde dem Bundesblatt vom 16. März 1872 beigelegt (BBl 1872 I 504, 858), ist jedoch über das Internet nicht greifbar.

18 BBl 1872 II 358.

19 BBl 1873 II 774.

20 Bundesbeschluss vom 31. Januar 1974; Botschaft vom 20. Mai 1974 betreffend die Abstimmung vom 9. April 1874 über die abgeänderte Bundesverfassung, BBl 1874 I 699. Der Text der Verfassungsvorlage vom 31. Januar 1874 wurde dem Bundesblatt vom 14. Februar 1874 beigelegt (BB1 1874 I 1231), ist jedoch über das Internet nicht greifbar. tionalrat beschloss als Erstrat die Rückweisung der Vorlage an den Bundesrat, was er nach dem damals geltenden Recht ohne Zustimmung des Ständerates tun konnte ${ }^{21}$, und der Sache wurde keine weitere Folge mehr gegeben.

3. Im Dezember 1958 reichte die Sozialdemokratische Partei der Schweiz ein Volksbegehren auf Einführung der Gesetzesinitiative ein. Die Initiative scheiterte jedoch in der Volksabstimmung vom 22. Oktober 1961 deutlich 22 .

4. Im Zusammenhang mit der Totalrevision der Bundesverfassung von 1999 unterbreitete der Bundesrat den eidgenössischen Räten eine separate Vorlage über die Reform der Volksrechte ${ }^{23}$. Dieses «Paket» enthielt auch die Einführung einer allgemeinen Volksinitiative; es wurde aber von National- und Ständerat durch Nichteintretensbeschlüsse beiseitegeschoben 24 . Gleichzeitig mit seinem Nichteintretensbeschluss hiess der Ständerat immerhin eine parlamentarische Initiative gut, welche darauf abzielte, die Reform der Volksrechte nachzuholen. Der Vorstoss führte zu einem Vorschlag auf Einführung der allgemeinen Initiative durch Revision der Art. 138 ff. BV vom 4. Oktober 2002²5; dieser wurde in der Volksabstimmung vom 9. Februar 2003 mit grossem Mehr ange-

${ }_{21}$ Vgl. Botschaft vom 6. März 1906 betreffend Einführung der Volksinitiative für die Bundesgesetzgebung, BBl 1906 III 4 ff.; StenBull N 1906 1330, StenBull S 1907 I 170.

22 BBl 1960 I 361; BBl 1961 I 1172.

23 Botschaft BV 35 f., 436 ff., $635 \mathrm{ff}$.

24 AB N 19991021 f.; AB S 1999 609, 398 ff.

25 BB1 20026485. 
nommen ${ }^{26}$. Der Bundesrat unterbreitete dem Parlament darauf am 31. Mai 2006 eine Botschaft «über die Einführung der allgemeinen Volksinitiative und über weitere Änderungen der Bundesgesetzgebung über die politischen Rechte» mit Entwürfen zu einem BG über die Einführung der allgemeinen Initiative, einem BB über das vollständige Inkrafttreten der Änderung der Volksrechte vom 4. Oktober 2002 sowie einem BG betreffend die Änderung der Bundesgesetzgebung über die politischen Rechte (Vote électronique)27. National- und Ständerat traten auf die beiden ersten Entwürfe nicht ein, weil die vorgeschlagene Regelung zu kompliziert sei ${ }^{28}$. In der Folge wurden am 27. September 2009 die am 9. Februar 2003 angenommenen Verfassungsänderungen wieder aufgehoben ${ }^{29}$.

Das Bundesrecht hat somit, wie bereits unter II. dargelegt, das Institut der Initiative seit jeher nur auf der obersten Ebene der Rechtsetzung, d.h. als Verfassungsinitiative gekannt; die wiederholten Bemühungen um die Einführung von Volksbegehren für untere Rechtsetzungsstufen waren nie erfolgreich. Die Bedeutung der Unterscheidung zwischen Verfassungsrecht und unterstufigem Recht

\footnotetext{
BB1 20033111.

BBl 2006 5261, 5331, 5341, 5343.

AB N 2006 1979; AB S 2006225.

Häfelin/Haller/Keller Rz 1798-1799; Bericht der Staatspolitischen Kommission des NR vom 21. Februar 2008 (BB1 2008 2891); Stellungnahme BR vom 16. April 2008 (BBl 2008 2907); BB über den Verzicht auf die Einführung der allgemeinen Volksinitiative vom 19. Dezember 2008 (BB1 2009 13); BRB vom 1. Dezember 2009 über das Ergebnis der Volksabstimmung vom 27. September 2009 (BB1 2009 8719).
}

im Zusammenhang mit Volksbegehren war somit allen Beteiligten - mit Einschluss von Volk und Ständen - seit jeher bewusst.

\section{Die Praxis der Bundesversamm- lung bei der Prüfung von Volksbe- gehren}

\section{A. Prüfung nach Art. 121 aBV (bis 1999)}

15 Art. 121 Abs. 3 und 4 aBV normierten die Grundsätze der Einheit der Materie und der Form; die Kompetenz zur Prüfung von Volksbegehren wurde jedoch auf Gesetzesebene geregelt: Gemäss dem BG über das Verfahren bei Volksbegehren auf Revision der Bundesverfassung (Initiativgesetz) vom 23. März 196230 (Art. 3) wie nach dem BG über die politischen Rechte (BPR) vom 17. Dezember $19763^{11}$ (Art. 75) hatte die Bundesversammlung Volksinitiativen, welche gegen Art. 121 Abs. 3 und $4 \mathrm{aBV}$ verstiessen, ungültig zu erklären. Als weiterer - selbstverständlicher und deshalb ungeschriebener - Ungültigkeitsgrund wurde die praktische Undurchführbarkeit einer Initiative anerkannt 32 .

16 Unter der aBV erklärte die Bundesversammlung insgesamt vier Volksbegehren als ungültig, nämlich

3о $\mathrm{AS} 1962789$.

31 SR 161.1; das BPR hob das Initiativgesetz auf den 1. Juli 1978 auf (Art. 89 lit. c).

32 Botschaft BV 433, 438, 445; L. Wildhaber, Komm. aBV, Rz 111-118 zu Art. 118; A. Lombardi, a.a.O., Rz 9-10 zu Vorbem. zu Art. 138-142; Häfelin/Haller/Keller, Rz 1758-1759; É. Grisel, in: D. Thürer, J.-F. Aubert, J.P. Müller (Hrsg.), Verfassungsrecht der Schweiz, 2001 (zit. Grisel, Verfassungsrecht), $\S 24 \mathrm{Rz} 24$; ders., Initiative et référendum populaires, Traité de la démocratie directe en Suisse, 2004 (zit. Grisel, Initiative), Rz 647-654. 
1. die Volksinitiative «Vorübergehende Herabsetzung der militärischen Ausgaben (Rüstungspause)» vom 2. Dezember 1954 am 15. Dezember 1955 wegen objektiver Undurchführbarkeit33;

2. die Volksinitiative «gegen Teuerung und Inflation» vom 29. Mai 1975 am 17. Dezember 1977 und diejenige «für weniger Militärausgaben und mehr Friedenspolitik» vom 24. September 1992 am 20. Juni 1995, weil sie das Erfordernis der Einheit der Materie nicht erfüllten34;

3. die Volksinitiative «für eine vernünftige Asylpolitik» vom 6. Juli 1992 am 14. März 1996 wegen Verletzung zwingender Bestimmungen des Völkerrechts 35 .

Die Verfassungswürdigkeit des Inhalts von Initiativen gab in den ersten $63 \mathrm{Jah}-$ ren nach Einführung der Teilrevisionsinitiative immer wieder zu reden:

1. Das Parlament stellte bereits im $\mathrm{Zu}-$ sammenhang mit dem allerersten Volksbegehren «für ein Verbot des Schlachtens ohne vorherige Betäubung» (Schächtverbot) die Frage, ob eine Materie, die wie das Verfahren bei der Tötung von Schlachttieren «bloss polizeilich reglementarischer Natur» sei, in die Verfassung gehöre36.

2. Auch in den folgenden 60 Jahren wurde das Problem der Verfassungswürdigkeit des Inhalts von Volksbegehren mehrmals aufgegriffen37; zu

BBl 1955 II 1463.

BBl 1977 III 919; BBl 1995 III 570.

BBl 1996 I 1355.

StenBull N 27. März 1893420.

So hielt der Bundesrat z.B. in seiner Botschaft be- einer vertieften Diskussion kam es jedoch nie, und die Frage, ob sich die Zulässigkeit einer Prüfung von Volksbegehren auf die Verfassungswürdigkeit ihres Inhalts nicht als selbstverständlich aus Art. 121 Abs. 2 aBV ergebe, wurde vom Bundesrat und von der Bundesversammlung nie ernsthaft gestellt.

3. Erst die am 24. Februar 1953 eingereichte Volksinitiative «Schutz der Stromlandschaft und Verleihung Rheinau» (Rheinau-Initiative) veranlasste den Bundesrat, die Frage der Verfassungswürdigkeit von Volksbegehren ausführlich zu behandeln ${ }^{38}$. Die Rheinau-Initiative verlangte eine rechtlich nicht kontroverse Ergänzung von Art. 24bis Abs. 2 BV (Schonung und Erhaltung von Naturschönheiten) und - im Sinne einer Übergangsbestimmung - die Aufhebung der bereits 1944 erteilten Konzession für den Bau des Kraftwerks Rheinau. Der Bundesrat hielt in seinem Bericht mit Bezug auf den zweiten, rechtlich höchst umstrittenen Teil der Initiative zusammenfassend fest, die Bundesverfassung habe

«weder ausdrücklich noch stillschweigend materielle Schran-ken der Revision ... aufgestellt»,

und es stehe

«ausser Zweifel, dass in der Eidge-

treffend Einführung der Volksinitiative für die

Bundesgesetzgebung vom 6. März 1906 fest, es sei «rechtlich möglich, auf dem Wege der Partialrevision Bestimmungen, die zweifellos in Gesetze oder Verordnungen gehören würden, in die Bundesverfassung hineinzubringen» (BBl 1906 III 4).

38 Bericht BR an die Bundesversammlung über das Volksbegehren zum Schutze der Stromlandschaft Rheinfall-Rheinau vom 4. Mai 1954, BBl 1954 I 721 ff. (zit. Rheinau-Bericht). 
nossenschaft nur dem Volk und den Ständen die Befugnis zustehen kann, darüber zu ent-scheiden, was in die Verfassung aufgenommen werden kann und was nicht»39.

Den entgegengesetzten Meinungen hielt der Bundesrat entgegen, sie erklärten sich

«aus der Befürchtung, dass Volk und Stände demagogischen Einflüssen unterliegen und einen Entscheid treffen könnten, der den Staat und seine demokratischen Einrichtungen in ihren Grundlagen erschüttern könnte».

Wenn aber diese Befürchtungen begründet wären,

«hätte es wohl wenig Sinn, die Demokratie dadurch retten zu wollen, dass Volk und Ständen das Recht zur Willensäusserung in einer wichtigen Verfassungsfrage entzogen wird. Denn die Demokratie ist die Staatsform des Vertrauens in die Einsicht und den guten Willen des Volkes»40.

Die Stellungnahme des Bundesrates zur Gültigkeit der Rheinau-Initiative war offensichtlich vom Glauben an die Allmacht des Volkes geprägt; dies zeigt sich in einigen aus heutiger Sicht erstaunlichen Formulierungen, wie insbesondere, aus der Entstehungsgeschichte der aBV gehe hervor,

«dass man dem Volk und den Ständen die unbeschränkte Macht geben wollte, welche früher einem absoluten

39 Rheinau-Bericht 750.

40 Rheinau-Bericht $750 \mathrm{f}$. Die zitierte Passage wird neuestens von J.P. Müller/G. Biaggini (Die Verfassungsidee angesichts der Gefahr eines Demokratieabsolutismus, ZBl 116/2015 235) zitiert und mit dem Satz ergänzt: «Hoffen wir, dass solches Vertrauen auch heute gerechtfertigt sei» (250).
Monarchen zustand»41.

Die Auffassung der Landesregierung setzte sich auch im Parlament durch42: Die Rheinau-Initiative wurde Volk und Ständen im Dezember 1954 vorgelegt, scheiterte aber deutlich43.

18 In der Folge wurde unter der aBV die vom Bundesrat im Zusammenhang mit der Rheinau-Initiative vertretene Meinung nicht mehr ernsthaft in Frage gestellt, sondern mehrmals ausdrücklich bestätigt 44 .

\section{B. Prüfung nach Art. 139 BV (ab 2000)}

19 Im Zuge der BV-Totalrevision von 1999 befasste sich der Bundesrat in seiner Botschaft unter dem Titel «Entwicklung und Stand des Verfassungsrechts» mit der Frage der Verfassungswürdigkeit45; die Frage der inhaltlichen Prüfung von Volksinitiativen spielte jedoch nur mit Bezug auf das Völkerrecht eine wesentliche Rolle. Im Zusammenhang mit der Frage weiterer Revisionsschranken befasste sich die Botschaft nur mit der faktischen Undurchführbarkeit und mit Rückwirkungsklauseln; im Übrigen verwies sie auf die herrschende Meinung 46 .

41 Rheinau-Bericht 747.

42 StenBull N 1954 126, 157, 173, 301; StenBull S 1954 116, 144, 187.

43 BB1 1955 I 63.

44 Vgl. z.B. den Zweiten Bericht des BR an die Bundesversammlung vom 8. August 1955 über das Volksbegehren für eine vorübergehende Herabsetzung der Militärausgaben (Volksinitiative für eine Rüstungspause), BB1 1955 II 325, 340 («Initiative Chevallier»).

45 Botschaft BV 19.

46 Botschaft BV 361 f., 445 ff. Die Ausführungen waren Teil des Abschnitts «Reformbereich Volksrechte», welchen die eidgenössischen Räte in der Folge zunächst durch Nichteintretensentscheide beiseite schoben (vgl. III. hievor); B. Ehrenzeller/ 

kompetenz der Bundesversammlung mit Bezug auf Einheit der Form und der Materie sowie Verletzung zwingender Bestimmungen des Völkerrechts auf Verfassungsstufe (Art. 139 Abs. 3 i.V.m. Art. 194 BV) geregelt. Das BPR47 wiederholt in Art. 75 Abs. 1 die Regel von Art. 139 Abs. 3 BV und konkretisiert in Abs. 2 und 3 die Begriffe «Einheit der Materie» und «Einheit der Form».

Seit dem Inkrafttreten der geltenden Bundesverfassung am 1. Januar 2000 erklärte die Bundesversammlung einmal eine Volksinitiative wegen Verletzung zwingender Grund-sätze des Völkerrechts als teilweise ungültig48; die Ungültigerklärung eines Volksbegehrens mit unterstufigem Inhalt betrachten Bundesrat und Bundesversammlung nach wie vor als unzulässig 49 .

\section{Fazit}

22

Die Praxis des Parlaments läuft auf eine sehr einfache Formel für die Prüfung von Volksbegehren hinaus: Jede gültig zu-

A. Lombardi in B. Ehrenzeller (Hrsg.) et al.: Die schweizerische Bundesverfassung: Kommentar, 2002, Rz 8-10 Vorbem. zu Art. 136-142.

47 SR 161.1.

48 Vgl. Botschaft vom 20. November 2013 zur Volksinitiative «Zur Durchsetzung der Ausschaffung krimineller Ausländer (Durchsetzungsinitiative)» (BB1 20139459 ff.) 9467 ff. und BB vom 20. März 2015 über diese Initiative (BB1 $20152701 \mathrm{ff}$.).

49 Vgl. dazu z.B. die Botschaft vom 7. Juni 2004 über die Volksinitiative «Für einen zeitgemässen Tierschutz (Tierschutz - Ja!)» BB1 20043283 (3293 ff.); «Voraussetzungen für die Gültigkeit von Volksinitiativen und die materiellen Schranken der Verfassungsrevision», Bericht des Bundesrates vom 5. März 2010 über das Verhältnis von Völkerrecht und Landesrecht (BB1 2010 2263) und Zusatzbericht vom 30. März 2011 (BBl 2011 3613); Bericht des Bundesamtes für Justiz zuhanden der Kommission für Rechtsfragen des Nationalrates vom 28. Dezember 2006 (VPB 1/2012 55). stande gekommene Initiative, die weder wegen Verletzung von Art. 139 Abs. 3 BV ungültig noch objektiv undurchführbar ist, kommt ungeachtet ihres Inhalts zur Abstimmung und wird im Falle der Annahme durch Volk und Stände Bestandteil der Verfassung. Anders ausgedrückt wird Verfassungsrecht alles, was im Verfahren der Verfassungsrevision zustande gekommen, d.h. nach der herkömmlichen Auffassung formelles Verfassungsrecht ist. Ob der Inhalt eines Volksbegehrens auch materiell als Verfassungsrecht, d.h. als der staatlichen Grundordnung zugehörig und damit verfassungswürdig $\mathrm{zu}$ qualifizieren ist, spielt keine Rolle.

\section{Die Konsequenzen der bisherigen Prüfungspraxis in neuerer Zeit}

23 Der Zweck der Bestimmungen über die Teilrevisionsinitiative ist es, den Stimmberechtigten zu ermöglichen, unmittelbar (d.h. unter Ausschaltung des ordentlichen Rechtsetzungsverfahrens auf der Verfassungsstufe) einen Entscheid von Volk und Ständen über den Inhalt der Verfassung herbeizuführen. Der Bundesrat führte dazu in seiner Botschaft von 1891 zur Einführung der Teilrevisionsinitiative Folgendes aus:

«Eine ... Erfahrung aller Kantone, in welchen Partialrevision auf dem Wege der Volksinitiative angebahnt werden kann, beweist ..., dass diese demokratische Institution in praxi keineswegs zu zahlreichen, hastigen und unüberlegten Revisionsanregungen führt, und es ist kein Grund, anzunehmen, dass sie im Bunde in ihren Wirkungen sich anders gestalten werde»50.

5o Botschaft 1890460. 
24 Die Entwicklung seit dem Inkrafttreten des damaligen Art. 121 aBV zeigt, dass die vorstehend zitierte Annahme heute keinesfalls mehr zutrifft:

1. In den ersten 78 Jahren nach der Einführung der Teilrevisionsinitiative blieb die Anzahl der Abstimmungen über solche Volksbegehren relativ stabil; das Total von 48 verteilte sich ziemlich regelmässig (eine Ausnahme bildete nur das Jahrzehnt 1921-1930 mit 13 Abstimmungen). Von 19712010 war jedoch ein dramatischer Anstieg mit 23, 34, 39 und 37 Abstimmungen pro Jahrzehnt zu verzeichnen ${ }^{51}$, und im laufenden Jahrzehnt haben bis Mitte 2015 bereits nicht weniger als 30 Abstimmungen stattgefunden.

Der Anteil der Abstimmungen über Volksbegehren an der Gesamtzahl der Abstimmungen betrug für die Jahrzehnte von 1971-2010 28.4, 53.1, 36.8 und 46.3\%; für die ersten $4^{1 / 2} \mathrm{Jahre}$ des laufenden Jahrzehnts stieg dieser Faktor auf $71.4 \% 52$.

2. Betrachtet man die Anzahl der eingereichten Initiativen, so ergibt sich folgendes Bild53:

- Von 1892-1930 wurden total 27 Initiativen eingereicht; zwei Volksbegehren wurden zugunsten von Gegenentwürfen zurückgezogen. In den folgenden vier Jahrzehnten (1931-1970) waren es bereits 71 Volksbegehren, von denen aber nicht weniger als 36 zurückgezogen

51 Vgl. Kölz, NSVG II 916 f.

52 BA für Statistik (Link: BAS 1). Abstimmungen über eine Initiative und über einen Gegenvorschlag dazu sind nur einmal (als Initiativabstimmung) gezählt.

53 BA für Statistik (Link: BAS 2). wurden (6 zugunsten von Gegenentwürfen, 30 aus anderen Gründen); eine Initiative wurde ungültig erklärt.

- Von 1971-2000 wurden 144 Initiativen eingereicht; in 39 Fällen kam es zu Rückzügen (11 zugunsten eines Gegenentwurfs, 28 aus anderen Grün-den), und drei Initiativen wurden ungültig erklärt. Seit 2001 war die Einreichung von rund 90 Initiativen zu verzeichnen 54 .

- Ende Juni 2015 waren 14 Volksbegehren im Sammelstadium, 3 beim Bundesrat, 7 bei der Bundesversammlung hängig und 4 abstimmungsreif55.

25 Die Initiativflut ist zu einem wesentlichen Teil $5^{6}$ darauf zurückzuführen, dass Parteien und andere Interessengruppen in vielen Fällen durch Volksbegehren gar nicht Verfassungsrecht schaffen wollen, sondern die Initiative zum Instrument der Rechtsetzung auf der Gesetzesstufe entfremden oder zum reinen Propagandawerkzeug pervertieren. Die Erfolgs-aus-sichten sind besonders im letzteren Fall häufig Nebensache, wenn die Initianten nur ein möglichst grosses Medienecho (z.B. im Hinblick auf Wahlen) erzielen 57 .

54 Das Bundesamt für Statistik führt seine genaue Zählung seit 2003 nicht mehr weiter.

55 BA für Statistik (Link: BAS 3 und Folgeseiten 2-4).

56 Dass auch die Ausweitung der Bundeskompetenzen und die durch sie verursachte Zunahme der Gesetzgebungstätigkeit auf Bundesebene zum Anschwellen der Initiativflut beigetragen haben, wurde bereits eingangs gesagt.

57 Vgl. R. Stark: Initiativen vom Wühltisch, Basler Zeitung 28. November 2013 9, wo ein Mitglied des Ständerates im Zusammenhang mit der am 24. November 2013 abgelehnten Initiative «1:12 - für gerechte Löhne» wie folgt zitiert wird: «Es ist doch 
Die zeitliche Belastung von Verwaltung, Bundesrat und Bundesversammlung durch die verwaltungsinterne und parlamentarische Behandlung nicht verfassungswürdiger und z.T. geradezu absurder Volksbegehren ${ }^{8}$, die personelle und finanzielle Inanspruchnahme von Bund und Kantonen durch Vorbereitung und Durchführung der Abstimmungen sowie die Strapazierung der Stimmbürger durch die häufigen Urnengänge drohen zu einer Gefahr für das politische Leben der Schweiz zu werden: Die politische Hauptarbeit der Behörden leidet59, und die Staatsverdrossenheit der Stimmberechtigten steigt - eine auf die Dauer unheilvolle Entwicklung60.

normal, dass man eine Initiative macht, um ein Thema auf die politische und mediale Agenda zu setzen. Das ist gelungen. Jetzt ist der Fall aber auch erledigt. Nun geht es wieder um die konkrete politische Arbeit».

58 Vgl. beispielsweise die z.Z. im Sammelstadium befindlichen Initiativen «Höchstgeschwindigkeit $140 \mathrm{~km} / \mathrm{h}$ auf Autobahnen» und «Für die Würde der landwirtschaftlichen Nutztiere (HornkuhInitiative)» sowie die weiteren Beispiele bei Biaggini in: BV Kommentar, Bundesverfassung der Schweizerischen Eidgenossenschaft, 2007 (zit. Biaggini BV Komm.) Rz 4 zu Art. 139; Wildhaber, Komm. aBV, Rz 100 zu Art. 118.

59 Die Behandlung von Volksbegehren und Gegenvorschlägen belegte z.B. im Amtlichen Bulletin der Bundesversammlung 1996 noch rund 6,6\% der Protokollseiten; 2013 waren es rund 12,2\%.

$60 \mathrm{Vgl}$. dazu schon die Botschaft Erhöhung 134 f.: «Es steht ausser Zweifel, dass die gegenwärtige Massierung hängiger Volksinitiativen den politischen Entscheidungsprozess überfordert. Eine Überbeanspruchung der politischen Behörden und der Verwaltung, aber auch weiterer Beteiligter am Rechtsetzungsprozess, vor allem der Stimmbürger, ist unverkennbar. ... Ein Blick auf den Abstimmungskalender für die nächsten Monate und Jahre verstärkt den Eindruck, dass wir erst am Anfang einer Intensivierung der Beanspruchung insbesondere der Stimmbürger stehen, die bereits heute Anzeichen der Ermüdung zeigen. Die zunehmende politische Abstinenz bei wichtigen Vorlagen wird als Alarmzeichen gedeutet.»
27 Der steile Anstieg der Anzahl von Abstimmungen über Volksinitiativen ist jedoch nicht die einzige negative Konsequenz der bisherigen Prüfungspraxis. Noch weit gefährlicher ist ein Aspekt, welcher der Öffentlichkeit bis vor Kurzem kaum bewusst geworden ist. Auf diese Entwicklung ist im Folgenden näher einzugehen.

\section{Die Verschiebung der verfas- sungsmässigen Kompetenzord- nung}

\section{A. Der Stand der Diskussion über die Bedeutung der Verfassungswür- digkeit}

28 Der heutige Stand der Diskussion über die Bedeutung der Verfassungswürdigkeit und des Begriffs der materiellen Verfassung lässt sich wie folgt zusammenfassen:

1. In der Lehre wird nach wie vor zwischen der Verfassung im materiellen und im formellen Sinn unterschieden, jedoch die Auffassung vertreten, die Unterscheidung zwischen materiellem und formellem Verfassungsrecht sei «entbehrlich» bzw. «juristisch ganz und gar überflüssig» 6 . Dass die Verfassung nur Bestimmungen enthalten sollte, welche im formellen und im materiellen Sinn Verfassungsrecht, d.h. vom Verfassungsgeber erlassen worden und inhaltlich der staatlichen Grundordnung zugehörig sind, wird

61 R. Rhinow/M. Schefer: Schweizerisches Verfassungsrecht, 2. Aufl. (zit. Rhinow/Schefer) Rz 25 ff.; P. Tschannen, Staatsrecht der Schweizerischen Eidgenossenschaft, 2011 (zit. Tschannen) § $3 \mathrm{Rz} 7 \mathrm{ff}$;; bedeutend weniger weit gehen Häfelin/Haller/Keller Rz 1761. 
zwar kaum bestritten62, aber als rein rechtspolitisches oder gar «verfassungsästhetisches» Postulat abgetan ${ }^{63}$. Eine Mehrheit der Lehre teilt somit im Wesentlichen die von Bundesrat und Bundesversammlung unter Berufung auf die Volkssouveränität vertretene Auffassung, einzig Volk und Stände hätten zu bestimmen, was Bestandteil der BV werden könne ${ }^{64}$. Am weitesten geht wohl die 2014 erschienene dritte Auflage des St. Galler Kommentars zur BV (Hervorhebungen mit Ausnahme der ersten durch den Verfasser):

«Im Rahmen der Schrankennorm von Abs. 3 können die Initianten eine beliebige Revision der BV vorschlagen. Das schweizerische Verfassungsrecht kennt keinen materiellen Verfassungsbegriff (...). Das Kriterium der Verfassungswürdigkeit hat somit keine rechtliche Bedeutung, sondern ist vielmehr ein rechtspolitisches Postulat. Eine Volksinitiative darf auch Bestimmungen zum Gegenstand eines obligatorischen Referendums machen, die weniger wichtig sind und eher in ein Gesetz oder eine VO gehören würden. Daran hat auch die mehrfache explizite Ablehnung der Einfuihrung einer Gesetzesinitiative nichts geändert (...). ... Durch das Fehlen eines materiellen Verfassungsbegriffs entfällt zudem

$6 2 \longdiv { \text { Rhinow/Schefer Rz 30; Häfelin/Haller/Keller Rz } 2 2 }$ f.; Biaggini in Biaggini et al., S. 72, Rz 5; Tschannen $\$ 3 \mathrm{Rz} 16$.

63 Tschannen $\S 3 \mathrm{Rz} 16$.

64 Vgl. insbesondere Wildhaber, a.a.O., Rz 90 ff. zu Art. 118; J.-F. Aubert: Traité de droit constitutionnel suisse, 1967, Rz 333; Lombardi, a.a.O., Rz 5 f. Vorbem. zu Art. 138-142; Biaggini, in Biaggini BV Komm. Rz 4 zu Art. 139; Häfelin/Haller/Keller Rz 1755, 1761; Tschannen Rz 7, 16; Rhinow/ Schefer Rz 30, 117, 467. auch grundsätzlich der Rechtssatz als Kriterium für die Verfassungsgebung. Nicht nur generell-abstrakte Normen, auch Einzelakte können Gegenstand einer Volksinitiative sein ... ${ }^{65}$.

2. Die gegenteilige Auffassung, wonach die Gültigkeit einer Volksinitiative die Verfassungswürdigkeit ihres Inhalts voraussetze, wird insbesondere von É. Grisel vertreten, welcher in diesem Zusammenhang den Begriff «unité de rang» verwendet und ihn gleichberechtigt der «unité de forme» sowie der «unité de matière» an die Seite stellt:

«Le principe de l'unité de rang est implicite, mais ressort clairement $d u$ système établi par la Constitution. L'article 139 entraîne deux corollaires: linitiative populaire doit d'abord viser la Constitution et non un texte normatif de niveau inférieur; ensuite, elle ne saurait comporter à la fois des dispositions constitutionnelles et législatives. Ces limites, qui dépendent l'une de l'autre, se justifient à trois égards. Avant tout, l'article 195, combiné avec l'article 141, implique que la démocratie semi-directe avance en deux étapes: lorsqu'un projet exige une révision constitutionnelle, puis l'adoption d'une loi, ces opérations ne peuvent pas coïncider, elles doivent se dérouler successivement. L'unité de rang complète aussi celle de la matière: puisque l'ordre juridique distingue deux degrés de normes, il serait abusif de mélanger

65 B. Ehrenzeller/R. Nobs: St. Galler Kommentar zur Bundesverfassung, 3. Aufl. (zit. SG Komm. BV), Art. 139, Rz 19. 
les genres, car le peuple a le droit de se prononcer séparément sur les différents textes. Enfin et surtout, le régime semidirect suppose que le législateur ait son mot à dire après le vote du constituant; il serait déjoué, si la Constitution posait elle-même des règles légales.»66

\section{B. Die Bedeutung des Gewaltentei- lungsgrundsatzes für die Prüfung von Volksbegehren}

29 Die vorherrschende Meinung (VI. A. hievor) geht auf die im bundesrätlichen Rheinau-Bericht enthaltene Auslegung von Art. 118 aBV zurück67. Diese konzentriert sich auf die (auch in Art. 192 Abs. 1 BV enthaltene) Aussage, die BV könne «jederzeit ganz oder teilweise revidiert werden» und kommt zum Schluss, die BV enthalte weder ausdrücklich noch stillschweigend inhaltliche Schranken der Verfassungsrevision ${ }^{68}$. Ob diese Schlussfolgerung auch heute noch zutrifft, kann und muss im Rahmen der vorliegenden Untersuchung offen bleiben. Festzuhalten ist, dass die bundesrätliche Analyse in einem wesentlichen Punkt zu kurz greift:

3 o Der Rheinau-Bericht wirft die Frage, ob der Begriff «Bundesverfassung» in Art. 118 aBV bzw. in Art. 192 Abs. 1 BV nicht impliziere, dass eine Verfassungs-

66 Grisel, Initiative, Rz 622-623; vgl. auch Grisel, Verfassungsrecht, Rz 10, 18; M. Luchsinger: Die Prinzipien des Rechtsstaates als materielle Schranken der Verfassungsrevision, 1960, 44, 72, 121; P. Uebersax: Zur Zulässigkeit der Durchsetzungsinitiative - eine Einladung zur Reflexion, ZB1 115/2014 600 (609). Ehrenzeller/Nobs betrachten die Auffassung von Grisel ohne nähere Begründung als «mit der geltenden Ordnung nicht vereinbar» (a.a.O., Art. 139, Rz 19).

67 Vgl. dazu IV. A. hievor.

68 Rheinau-Bericht $740 \mathrm{ff}$. vorlage keine unterstufigen Rechtsnormen enthalten darf, ohne eingehende Begründung in den gleichen Topf wie die Frage nach den inhaltlichen Schranken der Verfassungsrevision und verneint sie ${ }^{69}$. Die beiden Fragen sind aber strikte zu trennen: Die Frage der Verfassungswürdigkeit einer Vorlage hat mit deren rechtlich-politischem Inhalt nichts $\mathrm{zu}$ tun; es geht nicht darum, wie ein bestimmtes Problem materiell geregelt werden soll, sondern um die Frage, ob die anvisierte Regelung auf der obersten Rechtsetzungsstufe zu treffen ist. Diese Frage steht in engem Zusammenhang mit der Einhaltung der verfassungsmässigen, gewaltenteiligen Zuständigkeitsordnung:

31 Gemäss der BV ist für den Erlass von Verfassungsnormen eine andere Instanz zuständig als für die Schaffung von unterstufigem Recht: Die Verfassungsgebung fällt in die Kompetenz von Volk und Ständen, während die Gesetzgebung unter Vorbehalt des fakultativen Referendums der Bundesversammlung obliegt; Bundesrat und Bundesverwaltung schliesslich sind zum Erlass von Verordnungsrecht zuständig (Art. 163-165 bzw. 182 BV $)^{70}$. Damit setzt die BV den Grundsatz der Gewaltenteilung ${ }^{71}$ um;

69 Dies mag darauf zurückzuführen sein, dass es bei der Rheinau-Initiative nicht um das Zusammentreffen von Normen der Verfassungs- und der Gesetzesstufe, sondern um die Gültigkeit einer Übergangsbestimmung in Form eines Konzessionswiderrufs ging.

70 Die Ausnahmen von dieser Regelung (z.B. Art. 159 Abs. 4, 163 Abs. 1 und 173 Abs. 1 lit. c BV) sind für die vorliegend angestellten Überlegungen nicht von Belang, weil sie das Verhältnis Bundesversammlung-Bundesrat betreffen. Im Folgenden ist der Einfachheit halber nur von Verfassungs- und Gesetzesrecht die Rede. Vgl. zur Frage der Gewaltenteilung insb. Uebersax (Fn. 66).

${ }_{71}$ Häfelin/Haller/Keller Rz 1410 ff. 
dieser muss in einem Staat, dessen Rechtsordnung je nach Erlassstufe die Mitwirkung unterschiedlicher Organe an der Rechtsetzung vorsieht, nicht nur auf das Verhältnis zwischen den drei Grundgewalten Legislative, Exekutive und Gerichtsbarkeit, sondern auch auf die Beziehung zwischen den unterschiedlichen rechtsetzenden Organen Anwendung finden ${ }^{72}$.

Die Verteilung der rechtsetzenden Gewalt auf den beiden obersten Stufen hat zur Folge, dass, wenn eine Verfassungsvorlage in Frage steht, die Rechtsetzung i.d.R. in zwei Phasen abzulaufen hat73:

1. Auf der Verfassungsstufe haben Volk und Stände über eine Vorlage zu entscheiden, welche

- entweder vom Parlament ausgegangen und durch dieses beraten worden ist;

- oder auf einem Volksbegehren beruht und vom Parlament nicht verändert, sondern nur durch einen Gegenvorschlag ergänzt werden darf.

2. Auf der Gesetzesstufe erfolgt die Konkretisierung der Verfassungsbestimmung durch das Parlament, dem unter Vorbehalt des fakultativen Referendums das Gesetzgebungsmonopol zusteht.

Das Verfahren der Rechtsetzung auf dem Weg der Volksinitiative ist grundlegend verschieden vom ordentlichen Rechtsetzungsverfahren74:

72 Gl. M. P. Uebersax (Fn. 66) 608.

73 Ausgenommen sind die - seltenen - Fälle, wo es möglich ist, eine Materie abschliessend auf der Verfassungsstufe zu regeln.

74 So wird im Folgenden das Rechtsetzungsverfahren
1. Am Anfang des ordentlichen Rechtsetzungsverfahrens steht in den meisten Fällen ein Erlassentwurf des Bundesrates, welcher zunächst in das Vernehmlassungsverfahren geht (Art. 3-5 VlG75). Nach Ablauf der Vernehmlassungsfrist werden die Stellungnahmen gewichtet und ausgewertet (Art. 8 VlG), und der Bundesrat verfasst seine Botschaft an das Parlament (Art. 141 ParlG76). Es folgt die Behandlung in den Kommissionen (soweit vorgesehen, Art. 42 ff. ParlG) und in den Plena von National- und Ständerat (Art. 71 ff. ParlG); untersteht die Vorlage dem obligatorischen Referendum oder kommt ein fakultatives Referendum zustande, so schliesst sich die Volksabstimmung an (Art. 140-142 BV).

2. Im Falle der Volksinitiative wird der gemäss Art. 69 BPR durch die Bundeskanzlei vorgeprüfte Initiativtext nach dem Zustandekommen des Volksbegehrens (Art. 72 BPR) durch den Bundesrat mit einer Botschaft der Bundesversammlung unterbreitet (bei einer Initiative in Form des ausgearbeiteten Entwurfs gegebenenfalls zusammen mit einem Gegenentwurf, Art. 97 ParlG). Das Parlament kann und muss die Initiative bei Vorliegen der Voraussetzungen ganz oder teilweise ungültig erklären (Art. 98 ParlG); ist die Initiative gültig, so hat die Bundesversammlung bei einer Initiative in Form des ausgearbeiteten Entwurfs zu beschliessen, ob sie das Volksbegehren zur Annahme oder Ab-

bezeichnet, das vom Bundesrat oder von der Bundesversammlung in Gang gesetzt wird (Art. $71 \mathrm{ff}$. ParlG).

75 SR 172.061.

76 SR 171.10. 
lehnung empfiehlt (Art. 100 ParlG). Die Abänderung eines gültigen Initiativtextes ist dem Parlament nicht gestattet (Art. 99 ParlG); es kann jedoch gleichzeitig mit der Initiative einen Gegenvorschlag zur Abstimmung unterbreiten (Art. 101 ParlG).

34 Der Unterschied zwischen den beiden Verfahren ist offensichtlich:

1. Das ordentliche Rechtsetzungsverfahren ist darauf angelegt, die Meinungen der interessierten Kreise schon vor Beginn der parlamentarischen Beratung kennenzulernen und zu berücksichtigen (Vernehmlassungsverfahren) sowie im parlamentarischen Verfahren Interessengegensätze durch das $\mathrm{Zu}-$ sammenwirken der beiden Kammern auszugleichen oder zu verringern.

2. Bei dem durch ein Volksbegehren eingeleiteten Rechtsetzungsverfahren muss die Initiative, soweit sie gültig ist, Volk und Ständen ohne jede Änderung unterbreitet werden (Art. 99 ParlG). Das Vernehmlassungsverfahren und, soweit nicht die Gültigkeit der Initiative betroffen ist, eine materielle Beratung durch National- und Ständerat entfallen.

Das Rechtsetzungsverfahren, das durch eine Initiative eingeleitet wird, schafft offensichtlich eine gewichtige Ausnahme zum ordentlichen Verfahren und liegt deshalb quer in der politischen Landschaft77. Das ist von der Verfassung gewollt, aber nur für Volksbegehren, welche auf eine Abänderung der Verfassung gerichtet sind. Die direkte Einflussnahme

77 Kley in Biaggini et al. § 24 Rz 68 f.; D. Moeckli: Die Teilungültigkeit und Aufspaltung von Volksinitiativen, ZBl 115/2014 598 (579). der Stimmberechtigten auf die unterstufige Rechtsetzung ist gemäss der Verfassung auf das Korrektiv des Gesetzesreferendums (Art. $141 \mathrm{f}$. BV) beschränkt78.

36 Eine Verfassungsvorlage, welche neben verfassungsrechtlichen auch unterstufige Normen oder ausschliesslich unterstufige Bestimmungen enthält, unterläuft die verfassungsmässige Kompetenzordnung gleich doppelt: Sie schränkt das Parlament in der Ausübung seiner Gesetzgebungskompetenz ein, und sie entzieht den Stimmberechtigten die Möglichkeit, auf dem Wege des fakultativen Referendums zum Resultat des Gesetzgebungsverfahrens Stellung zu nehmen. Die Konsequenz ist eine schleichende Verschiebung der verfassungsmässigen Zuständigkeiten durch eine Teilentmachtung des Parlaments und der Stimmberechtigten als Gesetzgeber.

37 Im Falle einer Volksinitiative mit unterstufigem Inhalt setzt letztlich eine durch das Initiativkomitee vertretene Interessengruppe mit der Formulierung des Initiativtextes die erwähnte Verschiebung der verfassungsmässigen Kompetenzen in Gang. Die Problematik dieser Entwicklung konnte der Bundesrat bei der Behandlung der Rheinau-Initiative vor rund 6o Jahren noch nicht voraussehen und würdigen; sie ist aber heute hoch aktuell, weil sich in neuester Zeit Volksbegehren häufen, welche

- durch extrem detaillierte Formulierung die Ausführungsgesetzgebung vorwegnehmen und dadurch in den Zuständigkeitsbereich des Gesetzge-

C. Schoch: Hybris des Volks beschädigt die Demokratie, NZZ 30. September 201411 a.E. 
bers eingreifen79;

- die «strikte» Umsetzung von angenommenen Volksbegehren verlangen, bevor die nach der Verfassung für die Umsetzung zuständige Behörde (i.d.R. die Bundesversammlung) ihre Arbeit abgeschlossen und gegebenenfalls das Volk über ein Referendum entschieden hat ${ }^{80}$.

38 Diese Entwicklung lässt sich nach der hier vertretenen Auffassung nicht einfach durch die Berufung auf die Volkssouveränität rechtfertigen; sie zeigt vielmehr, dass die vom Bundesrat im RheinauBericht vertretene Auffassung, nur Volk und Stände hätten zu entscheiden, was in die Verfassung aufgenommen werden kann $^{81}$, zumindest aus heutiger Sicht nicht mehr haltbar ist. Bereits unter der aBV hat Wildhaber 1988 festgehalten, der Glaube an die Allmacht des Volkes widerspreche «dem Mindestmass an Skepsis», das man «im liberalen Verfassungsstaat jeder Manifestation öffentlicher Gewalt entgegenbringen» sollte 82 . In neuester Zeit weisen J.P. Müller/ G. Biaggini mit Recht darauf hin, dass die BV nirgends von Volkssouveränität oder davon spreche, dass «das Volk» souverän

$79 \overline{\text { Ein Beispiel ist die Volksinitiative «gegen die Ab- }}$ zockerei», die am 3. März 2013 angenommen wurde (BB1 2013 3129).

8 Vgl. die Volksinitiative «Zur Durchsetzung der Ausschaffung krimineller Ausländer (Durchsetzungsinitiative)», welche die Umsetzung der am 28. Oktober 2010 angenommenen und in Kraft getretenen Initiative «für die Ausschaffung krimineller Ausländer (Ausschaffungsinitiative)» anstrebt und von der Bundesversammlung mit Ausnahme einer Bestimmung über das zwingendeVölkerrecht für gültig befunden wurde (BBl 2015 2701).

81 Rheinau-Bericht 750.

82 A.a.O., Rz 121 zu Art. 118; vgl. auch J.P. Müller/ G. Biaggini (Fn. 40); C. Schoch (Fn. 78); D. Binswanger: Falscher Volksentscheid, Das Magazin 41/20146. sei; Gewaltenteilung und Schutz individueller Freiheit setzten auch der Verfügungsgewalt des Volkes klare Grenzen ${ }^{83}$.

\section{Der materielle Gehalt des Begriffs «Bundesverfassung»}

39 Die bisherige Praxis der Prüfung von Volksinitiativen läuft darauf hinaus, dem Begriff «Bundesverfassung», wie er in Art. 139 und 194 BV verwendet wird, jeden materiellen Gehalt und damit dem Kriterium der Verfassungswürdigkeit von Volksbegehren jede rechtliche Bedeutung abzusprechen. Nach der hier vertretenen Auffassung hat jedoch bei jeder Interpretation einer Rechtsnorm deren materieller Gehalt im Vordergrund zu stehen. Warum dies ausgerechnet für grundlegende Verfassungsnormen wie diejenigen über die Verfassungsrevision nicht gelten sollte, ist nicht einzusehen; gerade hier dürfen sich die zuständigen Behörden der Verpflichtung nicht entziehen, den materiellen Gehalt des Be-griffs «Bundesverfassung» zu ermitteln. Dass dies mit Schwierigkeiten verbunden sein kann, ändert an der Verpflichtung nichts.

40 Behörden und Lehre haben sich seit langem - und auch in neuerer Zeit - immer wieder intensiv mit dem materiellen Begriff der Verfassung auseinandergesetzt:

1. In der Botschaft zur Totalrevision der Bundesverfassung vom 20. November 1996 hat der Bundesrat unter dem Titel «Verfassungsrang» die Grundzüge der Unterscheidung zwischen Verfassungsrecht und unterstufigem Recht wie folgt formuliert ${ }^{84}$ :

83 Fn. 40 (238-240).

84 Botschaft BV 19; vgl. auch Rhinow/Schefer Rz 27, $471 \mathrm{f}$. 
«Verfassungswürdig sind zweifellos Bestimmungen, die in allgemeiner Weise das Wesen der Schweizerischen Eidgenossenschaft umschreiben. Verfassungswürdig sind sodann Bestimmungen, die die grundlegenden Rechte und Pflichten der Bürgerinnen und Bürger nennen. $\mathrm{Zu}$ den verfassungswürdigen Bestimmungen gehören im weiteren namentlich solche, die die wesentlichen Ziele und Aufgaben des Staates festlegen. Unentbehrlich sind dabei in unserem föderalistisch organisierten Staatswesen die Bestimmungen über die Zuständigkeitsverteilung zwischen dem Bund und den Kantonen. Verfassungswürdigkeit kommt schliesslich vor allem auch den Bestimmungen über das institutionelle Grundgerüst des Staates und die wichtigsten Entscheidungsverfahren zu.»

Im Folgenden hat der Bundesrat jedoch die «Zeitbezogenheit» und die «politische Vereinbarung» der Verfassungsmaterie zum Anlass genommen, dem Kriterium der Verfassungswürdigkeit die rechtliche Bedeutung abzusprechen ${ }^{85}$.

2. Die Ansichten der Lehre über den materiellen Begriff und die Funktionen der Verfassung stimmen untereinander und mit den unter 1. zitierten Ausführungen des Bundesrates in den grossen Zügen überein ${ }^{86}$ :

Die Verfassung ist der «Inbegriff der Rechtsnormen ..., die als Grundlage der rechtsstaatlichen und demokratischen Staatsordnung

85 Botschaft BV 19.

86 Die nachfolgenden Zitate sind als Beispiele zu verstehen. in die Verfassung gehören; sie umfasst insbesondere die Grundsätze der gewaltenteiligen Staatsorganisation, die Grundrechte und die politischen Rechte, andere rechtsstaatliche $\mathrm{Ga}$ rantien und Grundsätze über die Staatsaufgaben»87.

Zur Verfassung zählt man

«alle Rechtssätze, die wegen ihres Gehalts als die Grundlage eines freiheitlichen, demokratischen und sozialen Bundesstaats angesprochen werden können»88.

Die Verfassung regelt

«im Wesentlichen die Grundlagen und Grundzüge der politischen Ordnung, weist den Staatsbehörden ihre Aufgaben zu, sorgt für Machtbegrenzung und enthält die bestimmenden grundlegenden Werte für die Rechtsgemeinschaft»89.

Die Verfassung ist

«l'ensemble des règles juridiques relatives à l'Etat. Mais même cette formule est trop large, il faut encore la raboter à l'aide d'une délimitation supplémentaire ... Toutes les règles juridiques relatives à l'Etat ne font pas partie de la Constitution, mais seulement celles qui sont importantes. Tel détail du droit électoral, telle précision sur le calcul de limpôt peuvent bien se rapporter à l'Etat, mais on ne peut pas les rattacher à la Constitution; ce serait donner à ce terme une extension contraire au langage courant. Il faudra donc le réduire par l'usage d'un adjectif additionnel. On a
87 Häfelin/Haller/Keller Rz 17.

88 Tschannen, § $3 \mathrm{Rz} 11$.

89 P. Uebersax (Fn. 66) 602. 
dit les règles importantes; pour réduire encore davantage, on pourrait dire les règles fondamentales.»90

41 Die Bundesverfassung im materiellen Sinn kann somit definiert werden als Inbegriff der Rechtsnormen, welche die Grundlage der demokratischen, freiheitlichen, rechts-, bundes- und sozialstaatlichen Staatsordnung bilden, d.h. insbesondere die Bestimmungen, welche

- die wesentlichen Ziele, Aufgaben und Behörden des Staates sowie die grundlegenden Rechte und Pflichten der Bürgerinnen und Bürger umschreiben;

- im Verhältnis zwischen Bund und Kantonen die Verteilung der Zuständigkeiten und die Verfahren für die Auflösung von Kompetenzkonflikten festlegen;

- die Verfahren für die Einsetzung der wesentlichen staatlichen Behörden bestimmen.

42 Verfassungswürdig und als Inhalt einer Teilrevisionsinitiative zulässig wären somit nur Bestimmungen, welche der vorstehenden Umschreibung entsprechen. Dass die Umschreibung unbestimmte Rechtsbegriffe verwendet, welche die Entscheidungsinstanz im Einzelfall zu konkretisieren hat, ist normal und kein Grund, der Entscheidung auszuweichen ${ }^{91}$.

90 J.-F. Aubert in D. Thürer, J.-F. Aubert, J.P. Müller (Hrsg.): Verfassungsrecht der Schweiz/Droit constitutionnel suisse, 2001, § $1 \mathrm{Rz} 5$.

91 Vgl. dazu VII. A. hienach.

\section{Fazit}

43 Die Realität der Initiativpraxis hat sich seit der Einführung der Teilrevisionsinitiative vor rund 125 Jahren grundlegend verändert. Wenn das Ziel der Verfassungsauslegung die Ermittlung des «aktuell gültigen, situations- und wirklichkeitsbezogenen» Rechts sein soll92, so sind die zuständigen Behörden verpflichtet, der Zweckentfremdung und Pervertierung des Initiativrechts entgegenzuwirken, soweit dies im Rahmen des geltenden Rechts möglich ist. Ein Mittel da$\mathrm{zu}$ ist die Anpassung der bisherigen Prüfungspraxis an die Anforderungen der Wirklichkeit durch die Anerkennung der Verfassungswürdigkeit als Kriterium für die Gültigkeit von Volksinitiativen93.

\section{Einwände gegen die Prüfung auf Verfassungswürdigkeit}

44 Gegenüber der Prüfung von Verfassungsvorlagen auf die Verfassungswürdigkeit ihres Inhalts wurden und werden Einwände erhoben, auf welche im Folgenden einzugehen ist.

\section{A. Das Fehlen eines Begriffs der ma- teriellen Verfassung}

45 Dass kein Begriff des materiellen Verfassungsrechts existiert94, trifft nur insoweit $\mathrm{zu}$, als eine positivrechtliche Umschreibung des Begriffs in der Bundesverfassung gemeint ist. Das Fehlen einer Legaldefinition ändert aber nichts an der Tatsache, dass - wie in VI. B. hievor gezeigt - die Unterscheidung zwischen ma-

92 Rhinow/Schefer Rz 490.

93 Dass in Politik und Lehre ein Umdenken begonnen hat, wird unter IX. hienach ausgeführt.

94 Rheinau-Bericht 740 ff.; B. Ehrenzeller/R. Nobs in SG Komm. BV, Art. 139, Rz 19; Rhinow/Schefer $\mathrm{Rz} 30$. 
teriell verfassungsstufigem und materiell unterstufigem Recht für die schweizerische Rechtsordnung von grundlegender Bedeutung ist.

46 Der Einwand, eine Umschreibung der materiellen Verfassung bzw. des verfassungswürdigen Verfassungsinhalts sei untauglich, weil sie notwendigerweise unbestimmte Rechtsbegriffe enthalte, schlägt nicht durch:

Einmal gehört die Konkretisierung unbestimmter Rechtsbegriffe zu den alltäglichen Aufgaben jeder mit der Anwendung von Rechtsnormen befassten Behörde. Ausserdem sind der BV unbestimmte Rechtsbegriffe gerade im Zusammenhang mit der Abgrenzung zwischen verschiedenen Rechtsetzungsstufen keineswegs fremd 95 . Schliesslich enthält insbesondere Art. 139 BV noch andere Begriffe, deren Auslegungsbedürftigkeit nicht in Frage steht ${ }^{96}$.

Gemäss Art. 164 Abs. 1 BV sind «alle wichtigen rechtsetzenden Bestimmungen ... in der Form des Bundesgesetzes zu erlassen», und dazu gehören «die grundlegenden Bestimmungen» über die Ausübung der politischen Rechte etc. Der Bundesrat vertritt in der Botschaft BV (19) die Meinung, «mehr als die Hälfte der gegenwärtig hängigen oder angekündigten Verfassungsinitiativen könnte auf Gesetzesebene realisiert werden», woraus zu schliessen ist, dass auch die Unterscheidung zwischen verfassungsstufigem und unterstufigem Recht kein unlösbares Problem sein kann.

96 Dies gilt für alle drei in Art. 139 Abs. 3 BV als Ungültigkeitsgründe aufgeführten Begriffe. Vgl. dazu auch M. Schefer/A. Zimmermann: Materielle Schranken der Verfassungsgebung, LEGES 2011/3 343 (348). Im Übrigen war kein Geringerer als M. Imboden der Überzeugung, dass es möglich und richtig sei, die Verfassungswürdigkeit als Kriterium für die Zulässigkeit von Verfassungsvorlagen herbeizuziehen: Der 1958/59 unter seiner Leitung von Studenten der juristischen Fakultät der Universität Basel erarbeitete Verfassungsentwurf (Titel: «Die Bundesverfassung - wie sie sein könnte», enthalten in M. Imboden, Staat und Recht, 1971, 219 ff.)

\section{B. Der Wandel der materiellen Ver- fassung}

48 Es steht ausser Zweifel, dass der Begriff der materiellen Verfassung nicht ein- für allemal festgeschrieben ist, sondern sich im Laufe der Zeit wandelt97. Dies kann aber kein Argument gegen die Prüfung von Volksinitiativen auf die Verfassungswürdigkeit ihres Inhalts sein: Die Behörde, welche die Prüfung durchzuführen hat ${ }^{98}$, kann und muss ihre Prüfungspraxis veränderten Verhältnissen und Auffassungen ebenso anpassen, wie dies andere Behörden (Gerichte, Verwaltungsbehörden) zu tun haben. Dies gilt nicht nur für die Prüfung auf Verfassungswürdigkeit, sondern ebenso für die in Art. 139 Abs. 3 BV genannten Prüfungskriterien: Auch die Begriffe «Einheit der Form», «Einheit der Materie» und «zwingende Vorschriften des Völkerrechts» können sich wandeln, und sie sind, wie bereits erwähnt, ebenso auslegungsbedürftig wie der Begriff der materiellen Verfassung 99.

\section{C.Das Fehlen der Gesetzesinitiative}

49 Im Lauf der Jahrzehnte ist ein Argument gegen die Prüfung des Inhalts von Volksbegehren auf seine Verfassungswürdigkeit unzählige Male wiederholt worden:

formulierte den Grundsatz in Art. 58 Abs. 3 lapidar wie folgt: «Es können nur Bestimmungen in die Verfassung eingefügt werden, die sich auf die Grundordnung der Eidgenossenschaft beziehen.»

97 Häfelin/Haller/Keller Rz 1755, 1760-1762; Rhinow/Schefer Rz 25-30, 467; L. Wildhaber formuliert, die BV sei «weniger ... ein geschlossenes System objektiver Normen» als «ein in die Zeit offenes, mit der Zeit gehendes, elastisches Ordnungssystem» (Komm. aBV, Rz 120 zu Art. 118).

98 Siehe dazu Abschnitt X. hienach.

99 Häfelin/Haller/Keller Rz 1790 weisen z.B. mit Recht darauf hin, dass die Bundesversammlung 1995 ihre Praxis zum Grundsatz der Einheit der Materie verschärft hat. 
Weil die Rechtsordnung die Gesetzesinitiative nicht vorsehe, seien Initianten in vielen Fällen gezwungen, unterstufiges Recht in das Gewand einer Verfassungsinitiative zu kleiden ${ }^{100}$. Ein Mitglied des Nationalrates hat dies 2009 bei der Beratung der Volksinitiative «Für einen zeitgemässen Tierschutz (Tierschutz - Ja!)» wie folgt formuliert ${ }^{101}$ :

«Man kann nun ... allenfalls Recht geben, dass es eigentlich nicht etwas sei, was auf der Verfassungsstufe gelöst werden müsse. Wenn man aber wirklich findet, dass das nicht auf der Verfassungsstufe gelöst werden soll, dann hätte man zu einer vernünftigen Regelung im Rahmen der Strafprozessordnung oder im Rahmen des Tierschutzgesetzes Hand bieten müssen. Das haben Sie nicht gemacht, und von daher finde ich den Vorwurf von ... an die Vertreter des Tierschutzes eigentlich absurd, wenn sie das einzige Mittel ergreifen, das ihnen noch bleibt, nämlich eine eidgenössische Volksinitiative.»

Unverhohlener kann man das Pferd kaum am Schwanz aufzäumen: Die Teilrevisionsinitiative ist nicht dazu geschaffen worden, eine Vorlage der Gesetzesstufe, die im Gesetzgebungsverfahren keine Mehrheit findet, unter Umgehung des Parlaments als Verfassungsänderung durchzudrücken. Richtig ist vielmehr das Gegenteil: Gerade weil das geltende Recht die Gesetzesinitiative nicht vorsieht, ist es unzulässig, sie in Gestalt der

$100 \overline{\text { Botschaft vom 29. Januar } 1997 \text { zur Volksinitiative }}$ «für die 10. AHV-Revision ohne Erhöhung des Rentenalters» (BB1 1997653 ff.) 657 ff. Von einer Umgehung des Fehlens der Gesetzesinitiative spricht auch A. Kley in Biaggini et al. § $24 \mathrm{Rz} 64$.

101 AB N 20091150.
Verfassungsinitiative durch die Hintertüre zu praktizieren. Dies sollte eigentlich allerspätestens seit dem 27. September 2009 klar sein, als Volk und Stände die am 9. Februar 2003 gutgeheissene, aber nie in Kraft getretene Verfassungsänderung über die Einführung der allgemeinen Initiative wieder aufhoben ${ }^{102}$.

\section{D.Die Allmacht des Volkes}

51 Die vom Bundesrat in seinem Bericht vom 4. Mai 1954 zur Rheinau-Initiative vertretene Auffassung, nur Volk und Stände könnten entscheiden, was in die Verfassung aufgenommen werden kann (vgl. Fn. 38 ff.) ${ }^{103}$, ist aus heutiger Sicht nicht mehr haltbar. Auf die Äusserung von Wild-haber, der Glaube an die Allmacht des Volkes widerspreche «dem Mindestmass an Skepsis», das man «im liberalen Verfassungsstaat jeder Manifestation öffentlicher Gewalt entgegenbringen» sollte, wurde bereits hingewiesen ${ }^{104}$. J.P. Müller/G. Biaggini warnen in

102 Schon bald nach der Ablehnung einer Einführung der Gesetzesinitiative am 22. Oktober 1961 stellte der Bundesrat die Frage, ob Verfassungsinitiativen mit dem Inhalt von Gesetzesinitiativen in Zukunft noch als gültig anerkannt werden dürften (Bericht des Bundesrates vom 18. Juni 1962 an die Bundesversammlung über das Volksbegehren für das Entscheidungsrecht des Volkes über die Ausrüstung der schweizerischen Armee mit Atomwaffen, BB1 1962 II 18, 19 f.). Die Frage wurde jedoch offen gelassen, weil sich die Initiative nicht im Begehren um Änderung von geltendem Gesetzesrecht erschöpfte. Nicht nachvollziehbar ist für den Verfasser die apodiktische Auffassung von Ehrenzeller/Nobs, wonach auch die mehrfache explizite Ablehnung der Einführung einer Gesetzesinitiative nichts daran geändert habe, dass eine Volksinitiative auch Bestimmungen enthalten dürfe, die eher in ein Gesetz oder eine $\mathrm{VO}$ gehören würden

(SG Komm. Art. 139 Rz 19). Vgl. dazu auch M. Schefer/A. Zimmermann (Fn. 96) 603 f.; Uebersax (Fn. 66) $609 \mathrm{f}$.

103 Rheinau-Bericht 750.

104 VI. B. hievor; Schoch (Fn. 78); Binswanger (Fn. 82). 
neuester Zeit, dass die einzigartige Befugnis, über welche Volk und Stände mit der Verfassungsinitiative verfügen, in «eine neue Art von Absolutismus» abgleiten könne, und sie weisen zu Recht darauf hin, dass die neuen Möglichkeiten der Kommunikation nicht nur das Sammeln von Unterschriften erleichtert, sondern auch eine Intensivierung der Werbung und anderer Formen der Mobilisierung von Abstimmenden mit sich gebracht haben ${ }^{105}$.

\section{E. Fazit}

52 Zusammenfassend ergibt sich, dass die Einwände, welche gegenüber dem Kriterium der Verfassungswürdigkeit als Massstab für die Prüfung von Volksbegehren erhoben werden, nicht schlüssig sind. Dies gilt insbesondere für das nach der hier vertretenen Auffassung eher axiomatische Argument, die BV kenne keinen materiellen Verfassungsbegriff, und das Kriterium der Verfassungswürdigkeit habe deshalb keine rechtliche Bedeutung.

\section{VIII.Derogierendes Gewohnheitsrecht durch langjährige Prüfungspraxis?}

53 Die unter IV.-V. beschriebene Praxis der Bundesversammlung, Volksbegehren nicht auf die Verfassungswürdigkeit ihres Inhalts zu prüfen, war während mehr als 120 Jahren konstant. Wäre deshalb ein Festhalten an dieser Praxis mit der Begründung zu rechtfertigen, sie habe sich zum Gewohnheitsrecht verdichtet?

54 Die Bildung von Gewohnheitsrecht setzt zweierlei voraus: Eine langjährige, ununterbrochene und einheitliche Übung und eine durch sie geschaffene Rechtsüber-

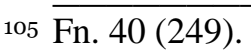

zeugung (opinio necessitatis) bei den rechtsanwendenden Behörden und bei den betroffenen Bürgern ${ }^{106}$. Das erste Erfordernis ist im vorliegenden Fall zweifellos erfüllt. Von einer Rechtsüberzeugung kann jedoch nicht die Rede sein, denn allzu zahlreich sind die Anzeichen dafür, dass den politischen Behörden die Fragwürdigkeit ihrer Prüfungspraxis von Anfang an sehr wohl bewusst war:

1. Bereits im Zusammenhang mit der allerersten Teilrevisionsinitiative (Schächtverbot) wurde 1893 in der Bundesversammlung die Frage aufgeworfen, ob die polizeiliche Regelung des Schächtens verfassungswürdig sei ${ }^{107}$.

2. In der Botschaft des Bundesrates betreffend Einführung der Volksinitiative für die Bundesgesetzgebung vom 6 . März 1906 wird ausgeführt ${ }^{108}$ :

« Es soll ... dem Volke die Möglichkeit gegeben werden, seinem Willen in geeigneter korrekter Form Ausdruck zu geben. Dass dies notwendig ist, beweist das Beispiel der Schächtinitiative. Es gibt keine andere Verfassung, die eine so eigenartige "Verfassungsbestimmung" wie den Schächtartikel (Art. 25 bis) der Bundesverfassung kennt» (Hervorhebung durch den Verfasser).

3. Im Bericht des Bundesrates an die Bundesversammlung «über ein Volksbegehren für die Einführung der Gesetzesinitiative im Bund» vom 29. Dezember 1959 steht unter dem Titel «Schutz der Reinheit der Verfassung durch die Gesetzesinitiative?» zu le-

\footnotetext{
106 Vgl. Häfelin/Haller/Keller Rz 12; BGE 81 I 34.

107 Vgl. z.B. StenBull N 1893420.

108 BBl 1906 III 4 f.
} 
sen 109:

"Initiantengruppen, die es anerkanntermassen gar nicht auf eine Verfassungsrevision ... absehen, sondern auf Erlass oder Änderung von Bestimmungen der Gesetzesstufe, greifen heute bisweilen zum Mittel der Verfassungsinitiative auf Partialrevision, indem sie sich darauf berufen, dass eben das passende Mittel, die Gesetzesinitiative, nicht zur Verfügung stehe. Hat eine solche Verfassungsinitiative Erfolg, so wird die Bundesverfassung mit Vorschriften belastet, die mit der Idee der Verfassung als Grundgesetz der Eidgenossenschaft nichts zu schaffen haben, sondern von Rechts wegen in ein einfaches Bundesgesetz gehören würden» (Hervorhebung durch den Verfasser).

4. In der nach Einführung des Frauenstimmrechts erstatteten Botschaft des Bundesrates «über eine Erhöhung der Unterschriftenzahlen für Initiative und Referendum» vom 9. Juni 1975 steht unter dem Titel «Materielle Schranken der Verfassungsinitiative» zu lesen ${ }^{110}$ :

"Im Vordergrund steht die Frage, ob schlechthin alles zum Gegenstand einer Verfassungsinitiative gemacht werden könne oder ob dieser inhaltliche (materielle) Schranken gesetzt seien. Während die politischen Bundesbehörden bisher solche Schranken verneint haben, gehen die Meinungen im schweizerischen Schrifttum weit

109 BB1 1960 II 363 f.

$110 \mathrm{Vgl}$. Botschaft Erhöhung 144, wo auch auf den Schlussbericht der Arbeitsgruppe für die Vorbereitung einer Totalrevision der Bundesverfassung vom 29. September 1972, S. 670 verwiesen wird. auseinander: Namhafte Staatsrechtler lehnen materielle Schranken vorbehaltlos ab, ebenso namhafte bekennen sich zur These, dass die Verfassung jedenfalls stillschweigende Schranken enthalte» (Hervorhebung durch den Verfasser).

5. Der Bericht des Bundesrates zur Totalrevision der Bundesverfassung (Motionen Obrecht und Dürrenmatt) vom 6. November 1996 hält fest ${ }^{111}$ :

«Die Verfassung enthält Normen, die nach verbreiteter Überzeugung der Verankerung in einem Staatsgrundgesetz nicht würdig sind und ihrer Bedeutung und Tragweite nach auf Gesetzes- oder gar Verordnungsstufe zu erlassen gewesen wären. Zu nennen sind etwa das Ordensverbot (Art. 12), das Absinthverbot (Art. 32ter) und der Spielbankenartikel (Art. 35). Zahlreich sind sodann die Bestimmungen, die - als Ergebnisse längst verglühter tages- und interessenpolitischer Hitzeschlachten - durch kaum erträgliche Weitschweifigkeit, Kompliziertheit, Unklarheit und Detaillierung auffallen» (Hervorhebung durch den Verfasser).

6. In der Botschaft zur Totalrevision der Bundesverfassung vom 20. November 1996 weist der Bundesrat wiederholt auf die Überwucherung der Verfassung durch inhaltlich nicht verfassungswürdige Teilrevisionsinitiativen hin ${ }^{112}$ :

«Eine wesentliche Lücke im System der Volksrechte ist das Feh-

111 BB1 1985 III 92.

112 Botschaft BV 439; der Begriff der Verfassungswürdigkeit erscheint in der Botschaft nicht weniger als 32-mal! 
len der Gesetzesinitiative. Mehr als die Hälfte der gegenwärtig hängigen oder angekündigten Verfassungsinitiativen könnte auf Gesetzesebene realisiert werden. Zwar wurde der Grossteil dieser Verfassungsinitiativen, die materiell Gesetzescharakter haben, verworfen, doch arbeitete die Bundesversammlung häufig Gegenentwürfe dazu aus, die in die Verfassung Eingang fanden, ohne dorthin zu gehören. Dadurch wurde die Verfassung von Details der Gesetzesstufe überwuchert. Und vor allem führt dies dazu, dass der Verfassungsgeber veranlasst wird, über normative Inhalte zu entscheiden, für die der Gesetzgeber - und zum Teil sogar der Verordnungsgeber rein funktionell betrachtet geeigneter wäre. Mit der Einführung der allgemeinen Volksinitiative könnte diese Lücke geschlossen werden» (Hervorhebung mit Ausnahme des ersten Satzes durch den Verfasser).

7. Im Zusammenhang mit der parlamentarischen Initiative «Beseitigung von Mängeln der Volksrechte», welche zur Verfassungsänderung von 2003 führte (vgl. II. hievor), äusserte sich die Staatspolitische Kommission des Ständerates in ihrem Bericht vom 2. April 2001 wie folgt ${ }^{113}$ :

«Volksbegehren, die auf Rechtsetzungsakte unterhalb der Verfassungsstufe oder auf Einzelakte abzielen, können heute nur auf dem Umweg über Verfassungsinitiativen eingebracht werden. Die allgemeine Volksinitiative oder das
Einzelaktreferendum würden zweckmässigere Verfahren schaffen» (Hervorhebung durch den Verfasser).

Die Kommission bezeichnete die allgemeine Volksinitiative als «Instrument, mit dem stufengerecht gesteuert werden kann» ${ }^{114}$, und Abs. 3 des in der Folge von Volk und Ständen angenommenen Art. 139a BV betreffend die allgemeine Volksinitiative hätte der Bundesversammlung denn auch ermöglicht, die für die Umsetzung der Initiative angemessene Rechtsstufe zu bestimmen ${ }^{15}$. Mit der Aufhebung der Verfassungsbestimmungen über die allgemeine Volksinitiative im September 2009 (vgl. II. hievor) fiel die Möglichkeit zur «stufengerechten Steuerung» der Rechtsetzung wieder dahin, ohne je in Kraft getreten zu sein.

55 Die vorstehenden beispielhaften Ausführungen ${ }^{116}$ liessen sich durch zahlreiche Äusserungen von Mitgliedern der Bundesversammlung ergänzen; sie zeigen, dass im Laufe der Jahrzehnte die Verfassungswürdigkeit des Inhalts von Teilrevisionsinitiativen immer wieder diskutiert und die Schaffung von unterstufigem Recht durch solche Volksbegehren kritisiert wurde. Dazu kommt, dass die Ein-

114 BB1 20014821.

115 Vgl. Botschaft vom 31. Mai 2006 über die Einführung der allgemeinen Volksinitiative und über weitere Änderungen der Bundesgesetzgebung über die politischen Rechte, BBl 20065261 (5266).

116 Weitere Beispiele finden sich in den Botschaften vom 29. Januar 1997 zur Volksinitiative «für die 10. AHV-Revision ohne Erhöhung des Rentenalters» (BBl 1997653 ff.) 657 ff. und vom 7. Juni 2004 über die Volksinitiative «Für einen zeitgemässen Tierschutz (Tierschutz - Ja!)» (BBl 20043283 ff.), wo der Bundesrat es als wesentlich erachtete, «die eidgenössischen Räte auf die problematische Regelungsstufe der ... Initiative hinzuweisen» (3295). 
führung der Gesetzes- bzw. der allgemeinen Initiative mehrmals versucht und verworfen wurde ${ }^{117}$ Eine für die Bildung von Gewohnheitsrecht notwendige Rechtsüberzeugung konnte sich so nicht entwickeln ${ }^{118}$.

\section{Neueste Entwicklungen}

56 Bis vor relativ kurzer Zeit sahen Politik, Lehre und Medien die Probleme des Initiativrechts vor allem im Missverhältnis zwischen Unterschriftenzahl und Anzahl der Stimmberechtigten sowie in der Entfremdung der Volksbegehren zum Mittel der politischen Propaganda. Die z.T. fragwürdigen Initiativen der letzten Jahre scheinen der Öffentlichkeit nun auch die Gefahr bewusst gemacht zu haben, welche eine schleichende Verschiebung der verfassungsgemässen Kompetenzordnung durch Initiativen mit unterstufigem Inhalt nach sich zieht. Demgemäss zeichnet sich in Lehre und Praxis ein Umdenken mit Bezug auf die hier untersuchte Frage ab:

1. Nachdem der Ständerat in der Sommersession 2014 die Initiative «Millionen-Erb-schaf-ten besteuern für unsere AHV (Erbschaftssteuerreform)» zur Prüfung der Gültigkeit an seine Kommission zurückgewiesen hatte ${ }^{119}$,

117 Vgl. III. hievor.

118 Unter diesen Umständen kann die Frage, ob sich Gewohnheitsrecht contra constitutionem überhaupt bilden kann (durch das Bundesgericht verneint in BGE 91 I 266; vgl. auch Häfelin/Haller/Keller Rz 12), offen gelassen werden.

119 BB1 2014 125; AB StR 3. Juni 2014, Votum V. Diener Lenz und Beschluss StR vom 3. Juni 2014. Die Gültigkeit wurde in der Folge bejaht (zu Recht, weil die Einführung einer neuen Bundessteuer zweifellos eine Verfassungsmaterie ist). In der Beratung wurde jedoch die Meinung vertreten, «dass aufgrund der Häufung von Volksinitiativen, begannen sich Politiker und Medien vermehrt zur Frage zu äussern ${ }^{120}$.

2. J.P. Müller/G. Biaggini ${ }^{121}$ machen die Möglichkeit von Konflikten zwischen Volks-souveränität und Gewaltenteilung deutlich ${ }^{122}$ und sprechen sich für die «Ein-bettung der Verfassungsinitiative in fundamentale Grundsätze eines demokratischen Staatsrechts» aus ${ }^{123}$. Besonderes Gewicht messen sie der Gewaltenteilung als Schranke der Volkssouveränität zu ${ }^{124}$.

3. R. Rhinow ${ }^{125}$ verweist unter dem Ein-

welche in Konflikt mit Grundrechten und grundlegenden Prinzipien unseres Rechtsstaates stehen, eine grundsätzliche Überprüfung der heute geltenden Kriterien für die Ungültigkeitserklärung von Volksinitiativen vorgenommen werden sollte» (AB StR 24. September 2014, Votum V. Diener Lenz). Das Volksbegehren wurde in der Abstimmung vom 14. Juni 2015 von Volk und Ständen verworfen.

$120 \mathrm{Vgl}$. z.B. R. Humbel, Das Parlament ist bei Initiativen zu grosszügig, Aargauer Zeitung 14. Juni 2014; G. Huber, Ist eine verschärfte Prüfung von Volksinitiativen nötig? (Link: Huber); K. Fontana, Immer dieser Ärger mit dem Volk, NZZ 14. Juni 2014 23; R. Zeller: Einstige Bundeskanzlerin will Initiativrecht einschränken, NZZ 2. November 2014 7; B. Neff: Wie ist der «Initiativflut» zu begegnen?, BaZ 4. November 2014 5; ders.: Volksinitiativen als Zumutung, BaZ 5. März 2015 2; M. Leuenberger: Demokratie als Ernüchterung, NZZ 9. März 2015 17; Sendung «Arena» des Fernsehens SRF «Darf das Volk alles?» vom 27. März 2015 (Link:Arena). Das von Avenir Suisse publizierte Diskussionspapier «Die Volksinitiative» (Verfasser: L. Rühli und T. Adler; Link: Avenir Suisse), welches einen Katalog von Vorschlägen zur Modifikation des Initiativrechts enthält, erwähnt auf S. 34 ff. die vorliegend behandelte Frage, kommt aber zum Schluss, dass eine Ungültigerklärung von Initiativen, die «zu sehr Gesetzescharakter» haben, «kaum dem (direkt-)demokratischen Selbstbild der Schweiz entsprechen» würde.

121 Fn. 40.

122 A.a.O. $241 \mathrm{ff}$.

123 A.a.O. 250.

124 A.a.O. $245 \mathrm{f}$.

125 R. Rhinow: Das getriebene Parlament: Volksinitiativen als mehrfache Herausforderung, Parlament, Parlement, Parlamento 1/15 25. 
druck der neuesten Entwicklungen u.a. auf

- die programmhafte, unsorgfältige und überschiessende Formulierung vieler Initiativen, die mehr auf die Funktionen eines permanenten Wahlkampfs als auf eine seriöse Einbettung neuere Normen in die BV zugeschnitten seien ${ }^{126}$;

- die Unsitte, Bestimmungen mit Gesetzescharakter auf die Stufe der Verfassung zu heben und dadurch das ordentliche Gesetzgebungsverfahren zu umgehen ${ }^{127}$;

- die seines Erachtens in der Wissenschaft unbestrittene Tatsache, dass es der Bundesversammlung nicht verwehrt ist, neue ungeschriebene Schranken des Initiativrechts zu anerkennen ${ }^{128}$.

Rhinow ruft die Bundesversammlung auf, ungeachtet allfälliger Reformen «von ihren verfassungsmässigen Kompetenzen klar und deutlich Gebrauch zu machen»; diese Äusserung könnte als Aufforderung zur Verschärfung der Praxis bei der Prüfung von Volksinitiativen verstanden werden ${ }^{129}$.

4. P. Uebersax ${ }^{130}$ nimmt die sog. Durchsetzungsinitiative ${ }^{131}$ zum Anlass, die Funktion von Verfassung und Gesetz sowie die von der BV festgelegten Or-

126 A.a.O. 25.

127 A.a.O. $26 \mathrm{ff}$.

128 A.a.O. 32.

129 Der zitierte Artikel von Rhinow vollzieht eine erstaunliche Kehrtwende gegenüber den vom Autor in Rhinow/Schefer (Rz 461 ff.) vertretenen Auffassungen; dies gilt in etwas geringerem Ausmasse auch für den Artikel von Schefer/Zimmermann (Fn. 96). 130 Fn. 66.

${ }_{131}$ Fn. 48. ganzuständigkeiten aufzuzeigen. Er kommt zum Schluss, dass der Bundesversammlung unter Vorbehalt des fakultativen Referendums ein verfassungsrechtliches Monopol auf die Gesetzgebung zusteht, und dass dieses Monopol durch Volksinitiativen verletzt wird, welche dem Parlament den Erlass der Ausführungsgesetzgebung zu einer bereits in der Verfassung als Gesetzgebungsauftrag geregelten Materie entreissen wollen ${ }^{132}$. Uebersax betrachtet es als fraglich, ob die Zulassung von verkappten Gesetzesinitiativen trotz dem Ausschluss dieser Initiativart auf Bundesebene mit der verfassungsrechtlichen Zuständigkeitsordnung vereinbar ist, und er warnt vor der Gefahr, dass sich «aus einer vorerst selektiven Aufweichung der $\mathrm{Zu}$ ständigkeitsordnung nach und nach eine neue Gewichtung der Kompetenzverteilung und damit der politischen Machtverhältnisse entwickeln könnte» ${ }^{333}$.

57 Es ist zu hoffen, dass sich diese Entwicklung nicht als Strohfeuer erweist, sondern die nötigen politischen Aktionen auslöst. Vor allem die Bundesversammlung sollte sich gegen ihre Entmachtung mit Nachdruck zur Wehr setzen.

\section{Zuständigkeit zur Prüfung von Volksbegehren}

58 Gemäss Art. 139 Abs. 3 BV ist die Bundesversammlung für die Ungültigerklärung von Volksbegehren wegen Verletzung der in der Verfassung ausdrücklich

132 A.a.O. $606 \mathrm{ff}$.

133 A.a.O. 610 f.; die Warnung ist berechtigt, weil bereits eine weitere «Durchsetzungsinitiative» angekündigt wurde. 
genannten Grund-sätze und Normen (Einheit der Form und der Materie sowie zwingende Bestimmungen des Völkerrechts) zuständig. Wenn Art. $139 \mathrm{BV}$, wie gezeigt wurde, die Prüfung von Volksinitiativen auch auf die Verfassungswürdigkeit ihres Inhalts zulässt, so kann keinem Zweifel unterliegen, dass für diese Prüfung ebenfalls die Zuständigkeit der eidgenössischen Räte giltt134.

59 Es ist hier nicht der Ort, die Frage zu beantworten, ob es richtig sei, wenn gerade die Bundesversammlung über die Verfassungswürdigkeit des Inhalts von Volksbegehren entscheidet ${ }^{135}$. Nach dem geltenden Recht ist die Zuständigkeit des Parlaments unzweifelhaft, und es liegt in seiner Verantwortung, die Prüfung so zu organisieren und durch-zu-führen, dass sie rechtlich solid und politisch glaubwürdig ausfälltt ${ }^{136}$. Es wäre bedenklich, wenn die Bundesversammlung eine Ausweitung der Prüfung von Volksbegehren auf die Verfassungswürdigkeit ihres Inhalts nur umsetzte, um politisch missliebige Initiativen besser $\mathrm{zu}$ Fall bringen zu können.

\section{Zusammenfassung und Ausblick}

60 Nach der hier vertretenen Auffassung ist das Kriterium der Verfassungswürdigkeit keineswegs ein rein rechtspolitisches

$134 \overline{\text { In diesem Sinne auch Wildhaber, Komm. aBV, Rz }}$ 116 zu Art. 121/122.

$135 \mathrm{Vgl}$. zu dieser Frage Ehrenzeller/Gertsch in SG Komm. zu Art. 139 BV, Rz 53; D. Moeckli (Fn. 77) 582; R. Rhinow (Fn. 125) 34 f.; M. Schefer/A. Zimmermann (Fn. 96) $357 \mathrm{f}$.

${ }^{136}$ In diesem Sinne der Bundesrat in der Botschaft BV 19 als Fazit der Ausführungen zur Frage des Verfassungsrangs: Die Verfassungswürdigkeit ist «nach einem möglichst einheitlichen, kohärenten Massstab zu beurteilen.»; vgl. auch Wildhaber, Komm. aBV, Art. 121/122 Rz 116.
Postulat ohne rechtliche Bedeutung ${ }^{137}$ oder gar ein blosses Postulat der Verfassungsästhetik ${ }^{138}$, sondern eine Schranke gegen die Überforderung der politischen Behörden mit Einschluss von Volk und Ständen durch die stetig wachsende Anzahl von Volksbegehren und insbesondere gegen die gefährliche schleichende Verschiebung der verfassungsmässigen Kompetenzordnung durch Volksinitiativen mit unterstufigem Inhalt. Deshalb ist es an der Zeit, dass die Bundesversammlung ihre bisherige Prüfungspraxis ändert und tut, wozu sie gemäss Art. 139 BV de lege lata befugt und verpflichtet ist: Volksinitiativen auf die Verfassungswürdigkeit ihres Inhalts prüfen und, wenn diese Prüfung negativ ausfällt, ungültig erklären.

61 Die Prüfung auf Verfassungswürdigkeit würde

- der staatspolitisch gefährlichen Entmachtung des Gesetzgebers durch materielles Gesetzesrecht steuern, das auf dem Weg der Verfassungsinitiative d.h. unter Umgehung des ordentlichen Gesetzgebungsverfahrens - geschaffen worden ist;

- die bisher durch Teilrevisionsinitiativen geschaffene unterstufige Spreu vom Weizen der Verfassungsstufe trennen und damit die übermässige Belastung von politischen Behörden und Stimmberechtigten durch nicht verfassungswürdige Volksbegehren reduzieren;

- längerfristig präventiv wirken, weil potentielle Initianten sich gut überlegen

137 Ehrenzeller/Nobs in SG Komm. zu Art. 139 BV, Rz 19.

${ }_{138}$ Tschannen, $\$ 3 \mathrm{Rz} 16$. 
müssten, ob sie die mit der Lancierung eines Volksbegehrens verbundenen Kosten und Mühen auf sich nehmen wollen, wenn sie die Ungültigerklärung eines Begehrens mit unterstufigem Inhalt riskieren ${ }^{139}$.

62 Die Vermischung von materiellem Verfassungs- und Gesetzesrecht durch Teilrevisionsinitiativen hat die Nachführung und Bereinigung der BV durch die Totalrevision von 1999 weitgehend zunichte gemacht und dem Respekt von Behörden und Stimmberechtigten vor dem höchsten Gesetz der Eidgenossenschaft geschadet. Die konsequente Trennung von Verfassungsrecht und unterstufigen Rechtsnormen durch die Erweiterung der Prüfung von Teilrevisionsinitiativen auf die Verfassungswürdigkeit ihres Inhalts würde auch dazu beitragen, die Achtung vor der Bundesverfassung, mit der es in den letzten Jahren nicht immer weit her gewesen ist ${ }^{140}$, wiederherzustellen.

139 Es läge im Interesse aller Beteiligten, möglichst früh zu erfahren, ob die Verfassungswürdigkeit eines geplanten Volksbegehrens zweifelhaft ist. Eine entsprechende (vorläufige) Beurteilung durch die Bundeskanzlei im Rahmen ihrer Vorprüfung würde eine Revision von Art. 69 BPR erfordern.

140 Als besonders krasses Beispiel sei hier die Kontroverse um das BG über die Buchpreisbindung (BuPG) erwähnt: Einer Interessengruppe, welche die Aufhebung der Buchpreisbindung durch die Wettbewerbskommission vor allen Instanzen erfolglos angefochten hatte, war es gelungen, die Bundesversammlung zum Erlass eines klar verfassungswidrigen Gesetzes zu bringen, das wegen des Ausschlusses der gerichtlichen Überprüfung von Bundesgesetzen (Art. 190 BV) verbindlich gewesen wäre. Das BuPG wurde jedoch in der Referendumsabstimmung vom 11. März 2012 abgelehnt. 